\title{
Intermittent bonding for high toughness/ high strength composites
}

\author{
A. G. ATKINS \\ Department of Mechanical Engineering, University of Michigan, 2046 East Engineering \\ Building, Ann Arbor, Michigan, USA
}

High strength and high toughness are usually mutually exclusive in brittle filament/brittle matrix composites. The high tensile strength characteristic of strong interfacial filament/matrix bonding can, however, be combined with the high fracture toughness of weak interfacial bonding, when the filaments are arranged to have alternate sections of high and low shear stress (and low and high toughness). Such weak and strong areas can be achieved by appropriate intermittent coating of the fibres. The strong regions ensure that the filament strength is picked up; weak areas randomly in the path of running cracks serve to blunt them by the Cook/Gordon mechanism which, in turn, produces long pull-out lengths with an associated large contribution to toughness. Boron-epoxy composites of volume fraction 0.20 to 0.25 have been made in this way which have fracture toughnesses of over $200 \mathrm{~kJ} \mathrm{~m}^{-2}$, whilst retaining rule of mixtures tensile strengths $(\sim 650$ $\mathrm{MN} \mathrm{m}^{-2}$ ). At the volume fractions used, this apparently represents $K_{\mathrm{IC}}$ values greater than $100 \mathrm{MN} \mathrm{m}^{-3 / 2}$.

An analysis is presented for toughness and strength which demonstrates, in broad terms, the effects of varying the coating parameters of concern. Results show that the "toughness" of interfaces is an important parameter, differences in which may not be shown up in terms of interfacial "strength". The choice of coating material is crucial in getting the desired effect.

Some observations are made upon methods of measuring the components of toughness in composites.

\section{Introduction}

Fracture in brittle matrix/brittle filament composites where the interfacial bond between fibre and matrix is strong often results in a fast matrix crack perpendicular to the filaments. Usually such an energetic crack breaks through all filaments in the path of the crack and complete fracture ensues. Even though shear debonding, of average length $l_{\mathrm{crit}} / 4$, may occur during fracture since the filaments will not necessarily break in the plane of a matrix crack, the associated "surfaces" contribution to toughness will be relatively limited because $l_{\text {crit }}$ is itself small [1]. Similarly, the contribution to toughness from Piggott/FitzRandolph stress redistribution $[2,3]$ is limited by the critical length, as is that from Cottrell/Kelly pull-out [4].

A general increase of $l_{\text {crit }}$ by lowering the filament/matrix shear bond will increase toughness, as discussed by Marston et al. [1]. In that (C) 1975 Chapman and Hall Ltd. paper, it was shown that a relationship between strength $(\sigma)$ and total composite toughness $(R)$ could be developed by recognizing that in general terms $R \alpha 1 / \tau$ where $\tau$ is the shear strength of the interfacial bond. In the case of the boron/ epoxy system for which data were presented in [1], the surface condition of the as-received $\mathrm{B} / \mathrm{W}$ filaments was such that when made up into composites with EPON-828 epoxy, strengths in the region of the rule of mixtures (RoM) value were attained. When the surface condition was altered by continuously coating the filaments with various substances, the strengths fell off and the toughness decreased (with strong interfaces), or increased (with weak interfaces). In the latter case, if the interfaces were sufficiently weak, there could be the possibility of introducing an additional contribution to toughness, namely Cook/Gordon tensile debonding ahead of the crack [5]. Such a mechanism, which is usually absent in conventional strongly bonded com- 
posites, may blunt and slow down cracks or arrest them completely.

However, weak interfaces throughout the composite can reduce the tensile strength quite significantly. Depending on the circumstances, perhaps $1 \mathrm{MN} \mathrm{m}^{-2}$ in tensile strength is "lost" for every $1 \mathrm{~kJ} \mathrm{~m}^{-2}$ "added" to toughness in laboratory testpieces; this follows from Equation 16 of [1] applied to boron/epoxy. The question that presents itself is whether there are any means by which the RoM tensile strength can be maintained along with high toughness values.

Marston [6] suggested that providing there were "enough" regions of high interfacial shear stress to ensure that the rule of mixtures strength was picked up, the rest of the composite could have quite weak interfacial bonds. Were such a composite to be laid up randomly with respect to weak and strong regions, both high strength and high toughness should be produced simultaneously. For if the lengths of the strongly bonded regions are greater than the critical length associated with that strong interfacial $\tau$, the filament strength would be attained, whilst at the same time, those weak interfaces situated randomly ahead of any running cracks would serve to blunt the cracks by debonding. The concept is shown schematically in Fig. 1.
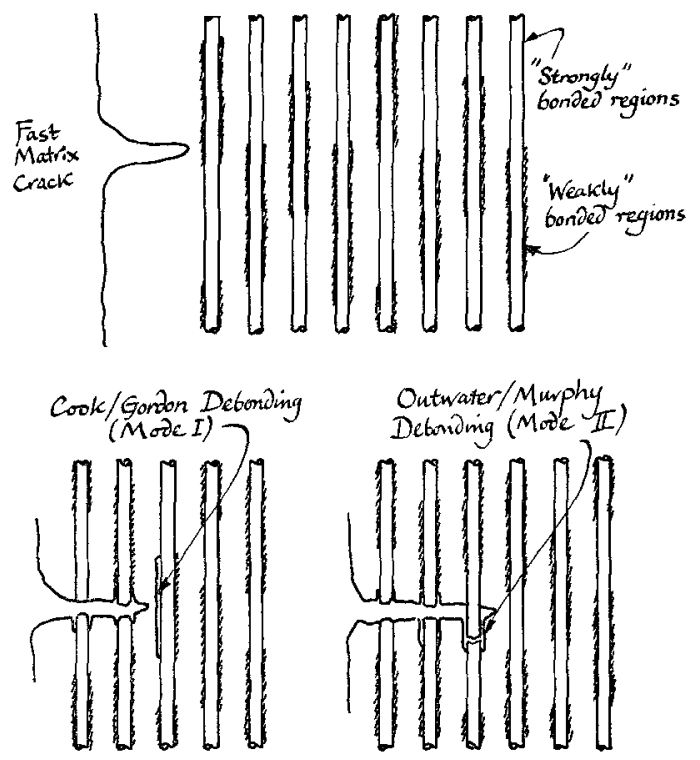

Figure 1 The intermittent bond and Cook/Gordon debonding.
Weakly and strongly bonded interfaces can be achieved by intermittently coating the filaments with some suitable substance before composite lay up.

How interfacial properties other than shear strength are affected by the coating procedure is an interesting and vital question, because it is probably the "toughness" of the interface that is of ultimate concern rather than the "strength". The tensile debonding envisaged by Cook and Gordon is fracture in mode I of fracture mechanics nomenclature*; the shear debonding implicit in the Outwater/Murphy analysis for toughness is fracture in the "forward sliding" mode II. The difference in modes was not clearly brought out in [1]. Each mode has its own toughness, $R_{\mathrm{I}}$ and $R_{\mathrm{II}}$, the explicit relationships of which to interfacial tensile and shear strengths are not obvious. Results for silicone vacuum grease (SVG) and polyurethane varnish (PUV) coatings are reported later in this paper. The uncoated regions have high interfacial shear stress and the coated regions are "weak", (being reflected in the relative transfer lengths). However, the SVG increased the toughness only modestly, whereas the PUV increases the toughness markedly; respectable tensile strengths were maintained with both coating materials. This emphasizes that the coating material is crucial, and revolves around the ill-understood interfacial toughness properties of the coatings.

An analysis for the strength and toughness of intermittently bonded brittle filament/brittle matrix composites is presented in the next section. The experimental results show quite strikingly that when appropriate coating materials are employed, strong, high toughness composites can be manufactured in the manner suggested [8].

\section{Mathematical model}

The following analysis is a modification of the treatment presented in [1]. Details of the derivations are given in the Appendix. The broad assumptions are that in a randomly laid up, intermittently bonded composite, the coated and uncoated regions of interfacial shear strengths $\tau_{\mathrm{c}}$ and $\tau_{\mathrm{uc}}$, may be represented by a rule of mixtures average shear strength $\tau_{a v}$ given by

$$
\begin{aligned}
\tau_{\mathrm{av}} & =(1-C) \tau_{\mathrm{ue}}+C \tau_{\mathrm{e}} \\
& =\tau_{\mathrm{uc}}\{1-C(1-T)\}
\end{aligned}
$$

\footnotetext{
*The Roman mode types are used here in the fracture mechanics Volterra dislocation sense, not in the nomenclature suggested by Mullin et al. [7], for different types of fracture observed in boron/epoxy systems. 
where $C=l_{\mathrm{c}} / l_{\text {r }}$, i.e. the ratio of coated length to repeat distance (see Fig. 2) and $T=\tau_{\mathrm{c}} / \tau_{\mathrm{uc}}$.

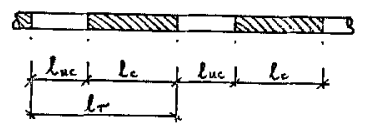

Figure 2 Intermittent bond geometry.

The "fictitious" critical length of the intermittently coated filaments is then given by

$$
\begin{aligned}
&\left(l_{\mathrm{crit}}\right)_{\tau_{\mathrm{av}}}=\frac{\sigma_{\mathrm{f}} d}{2 \tau_{\mathrm{av}}} \\
&=\frac{\left(l_{\mathrm{crit}}\right)_{\mathrm{uc}}}{\{1-C(1-T)\}} \\
&=\frac{\psi \lambda d}{\{1-C(1-T)\}}
\end{aligned}
$$

where $\sigma_{\mathrm{f}}$ is the filament tensile strength, and $d$ the filament diameter. The useful non-dimensional parameters are $\psi=\left(l_{\text {crit }}\right)_{\mathrm{uc}} / l_{\mathrm{r}}, \lambda=l_{\mathrm{r}} / d$.

In the same manner, we postulate the use of an average interfacial mode II toughness, to represent the behaviour of adjacent regions of low and high toughness, given by

$$
\begin{aligned}
\left(R_{\mathrm{IIif}}\right)_{\mathrm{av}} & =(1-C)\left(R_{\mathrm{IIif}}\right)_{\mathrm{uc}}+C\left(R_{\mathrm{II} \mathrm{if}}\right)_{\mathrm{c}} \\
& =R(\mathrm{II} \mathrm{if})_{\mathrm{uc}}\left[1-C\left(1-\rho_{\mathrm{II}}\right)\right]
\end{aligned}
$$

where $\left(R_{\mathrm{II} \text { if }}\right)_{\mathrm{uc}}$ and $\left(R_{\mathrm{II} \text { if }}\right)_{\mathrm{c}}$ are the uncoated and coated interfacial toughnesses, and $\rho_{I}$ is the ratio $\left(R_{\mathrm{II} \text { if }}\right)_{\mathrm{c}} /\left(R_{\mathrm{II} \text { if }}\right)_{\mathrm{ue}}$. It is arguable that this average should be weighted to take into account the instance of a crack growing in a non-bonded region, for example, and then propagating some distance into the well-bonded region before being arrested. The RoM approach has been taken for simplicity.

The foregoing type of toughness average is not used for mode I debonding, since cracking will not take place in the strong regions according to the Cook/Gordon model. The symbol $\rho_{\mathrm{I}}$ for $\left(R_{\mathrm{Iif}}\right)_{\mathrm{c}} /\left(R_{\mathrm{Iif}}\right)_{\mathrm{uc}}$ is, however, used later.

\subsection{Tensile strength}

For intermittently bonded filaments, the fibre load build-up may take place at low or high interfaces, or indeed over both types of interface, as shown schematically in Fig. 3. With the use of

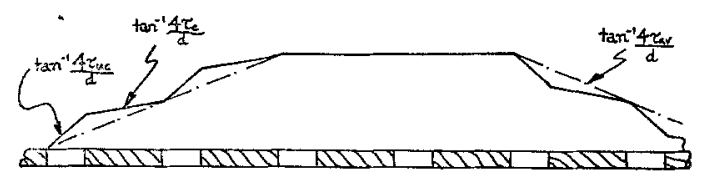

Figure 3 Critical shear transfer lengths. $\tau_{\mathrm{av}}$ to account for the different interfacial shear strengths, we can write for the RoM tensile strength

$$
\begin{aligned}
\sigma= & \left(1-v_{\mathrm{f}}\right) \sigma_{\mathrm{m}}+v_{\mathrm{f}} \sigma_{\mathrm{f}}\left[1-\frac{\sigma_{\mathrm{f}} d}{4 \tau_{\mathrm{av}} L}\right] \\
=\left(1-v_{\mathrm{f}}\right) \sigma_{\mathrm{m}} & \\
& \quad+v_{\mathrm{f}} \sigma_{\mathrm{f}}\left[1-\frac{\psi}{2 n\{1-C(1-T)\}}\right]
\end{aligned}
$$

where $v_{\mathrm{f}}$ is the filament volume fraction, $\sigma_{\mathrm{m}}$ the matrix tensile strength and $L$ the finite size of the testpiece or length of the filament. The nondimensional parameter $n$ is given by $L / l_{\text {r }}$.

For $C=0$, Equation 4 degenerates to the expression for continuously coated filaments [1]. At $C=1$ (and therefore $T=1$ ), the (lower) RoM tensile strength appropriate to the (lower) $\tau_{\mathrm{c}}$ is given. Note that at large $C$, the uncoated length is shorter than its own uncoated critical length, thus reducing its shear transfer potential, and thus contributing to the fall in $\sigma$ with $C$. It may be shown from Equation 1 that the value of $C$ at which this occurs is given by

$$
C>\frac{2-\psi}{2(1-T)}
$$

at which time more than one repeat distance is required to get the filament stress up to $\sigma_{\mathrm{f}}$ (Fig. 3 ). The upper bound to $\sigma$ is the uncoated RoM value itself, when load transfer happens to occur over regions of (large) $\tau_{u c}$, providing that they are as long as their own critical length.

\subsection{Fracture toughness}

According to [1] the total toughness, for continuously coated filaments, is given by

$$
R_{\text {total }}=R_{\text {surfaces }}+R_{\text {redist }}+R_{\text {pull-out }}
$$

where $R_{\text {surfaces }}$ related to debonding (mode II), $R_{\text {redist }}$ relates to Piggott/Fitz-Randolph stress redistribution (or relaxation), and $R_{\text {pull-out }}$ relates to Cottrell/Kelly pull-out.

An additional component, $R_{\text {Cook/Gordon, must }}$ be added to Equation 6 if tensile debonding ahead of the running crack takes place. It turns out that the Cook/Gordon mechanism itself is a comparatively small toughness sink; however, the associated additional debond lengths in the presence of Cook/Gordon debonding significantly increase the pull-out lengths and hence the total toughness. Using Equations 1 and 2, and other assumptions detailed in the Appendix, expressions may be obtained for the total 
toughness of intermittently bonded composites, by adding the various contributions from "surfaces", Piggott/Fitz-Randolph stress redistribution, Cottrell/Kelly pull-out and Cook/ Gordon mode I debonding (when acting). The final expression is rather cumbersome and is left to the Appendix. A physical picture of what governs each contribution is forthcoming in Section 4.

\section{Testpieces and experimental results}

Tensile and toughness specimens were made from layers of intermittently bonded epoxy composite tape manufactured on a drum apparatus with a device for coating the filaments before lay-up (Fig. 4). The tape is similar to Avco proprietary the repeat distances are not multiples of the drum circumference, alternate coated and uncoated regions are presented to a running crack; additionally test specimens consisted of many layers of tape, again helping the random lay-up concept. Tapes are stored in a refrigerator with the epoxy in the half-cured B-stage, complete curing $\left(12 \mathrm{~h}\right.$ at $120^{\circ} \mathrm{C}$ followed by oven cooling) taking place after specimens consisting of various layers have been made.

To investigate the intermittent bond analysis, tapes were manufactured for a range of values of $\psi, \lambda$ and $C$. The principal coating materials were silicone vacuum grease (as used by Marston [6] in his preliminary experiments) and polyurethane varnish. The repeat distances $\left(l_{\mathrm{r}}\right)$ were

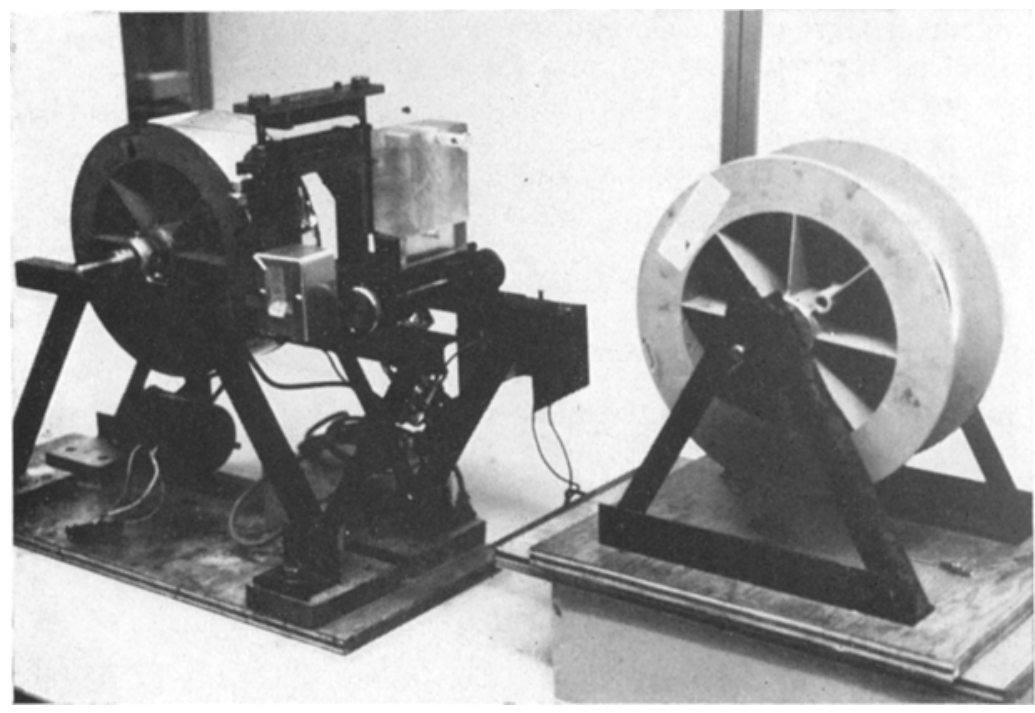

Figure 4 Photograph of coating and tape making apparatus.

"Rigidite, Prepreg" tape* except that the commercial tape has a filament surface condition that is uniform throughout. Our tape consists of a $250 \mu \mathrm{m}$ monolayer of $\mathrm{B} / \mathrm{W}$ filaments in EPON 828 epoxy, backed, for ease of handling, on 760 mm wide nylon scrim cloth of thickness about 50 $\mu \mathrm{m}$. The tapes are laid up on the periphery of a drum, the volume fraction of filaments being varied by altering the wrapping rate. The coating device is pneumatically operated, and "crimps" the filament with coating material, the coated/uncoated lengths being altered by the frequency of operation. The arrangement clearly does not give a truly random lay-up, but when variously $19,25.4$ and $51 \mathrm{~mm}$ which gave $0.05 \sim \psi \sim 0.13$, and $135 \sim \lambda \sim 360$. Independent tensile tests of $100 \%$ coated specimens suggested that $T=0.06$ (silicone vacuum grease) and $T=0.05$ (polyurethane). The toughness ratios $\rho$ were not known independently, but values can be inferred from the experimental data, as discussed later.

Tensile and toughness specimens were made from the same tape for a given combination of parameters. The tensile specimens consisted of 2 layers of the tape, in $100 \mathrm{~mm} \times 6 \mathrm{~mm}$ strips, with end tabs reinforced by additional layers of tape. Marston et al. [1] measured composite

*AVCO Systems Division, Lowell, Mass., USA. 
toughnesses using Tattersall and Tappin threepoint bending "roof" specimens [9]. To make comparison with the earlier data, some $5 \mathrm{~mm} \times$ $5 \mathrm{~mm} \times 35 \mathrm{~mm}$ Tattersall and Tappin specimens were made up from the tapes, but for reasons explained later, most toughness measurements in the present series were made on flat sheet edge-crack specimens, akin to ASTM "compact tension" specimens in profile [10]. These consisted of ten layers of tape in $76 \mathrm{~mm} \times 76$ $\mathrm{mm}$ panels as shown in Fig. 5. Originally it was

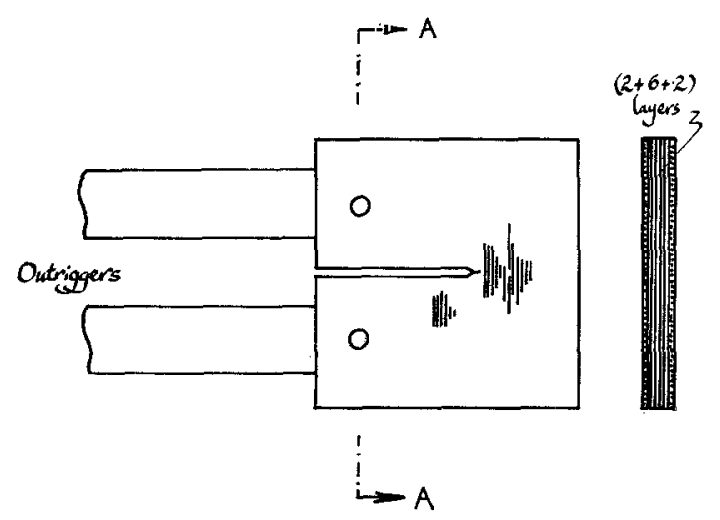

Figure 5 Edge-crack fracture toughness specimen.

intended to load the specimens at section AA, but bearing failures occurred at the holes and the crack velocities did not allow easy visual tracking in the less tough testpieces. Stcel outriggers were added to the specimens, which reduced both the crack load and the crack velocity. To prevent the composite arms above the crack from shearing off under load, two outside layers of tape on each side of the specimens were arranged with the filaments parallel to the crack. The central core of the specimen thus consisted of six unidirectional filaments perpendicular to the starter crack, where, within the limitations of the specimen and tape preparation method, the coated and uncoated layers occurred randomly relative to each filament.

Fracture toughness in the edge crack specimens was measured for increments of crack area, using Gurney's sector area technique [11] shown in Fig. 6 (the crack length being monitored during every test and the testing machine load extension trace "pipped" accordingly. Since Gurney's method does not involve a "compliance calibration", the alteration of specimen compliance, caused by the out-riggers, is inconsequential. Separate tests showed that the

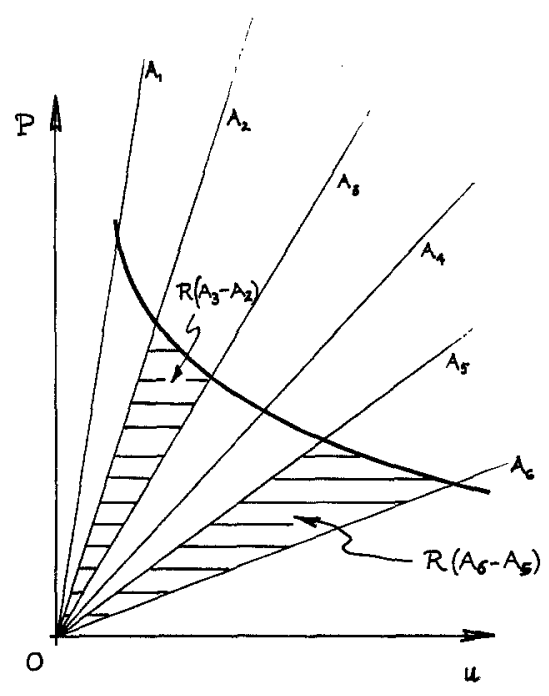

Figure 6 Gurney's sector area method for $R$. Radial lines from the origin represent constant crack areas (or lengths). During propagation, the fracture work required to advance the crack from area $A_{2}$ to $A_{3}$, for example, is given by the hatched area, of magnitude $R\left(\mathrm{~A}_{3}-\mathrm{A}_{2}\right)$, where $R$ is the fracture toughness.

matrix work of fracture in the outside layers of tape did not contribute significantly to the toughness. The load/extension plots showed some curvature before crack propagation especially in the high percentage coated samples. Upon unloading, after some crack propagation, the tougher testpieces showed marked "displacement irreversibility", i.e. the specimen cracks remained "open". Although geometric interference of filaments still bridging the crack obviously contributed to this effect, the question arises as to whether generalized yielding has occurred. Irreversibility at regions remote from the crack faces is a bane of fracture toughness testing of high toughness/low strength solids. If specimens are saw-cut along the crack into virgin material beyond the crack tip "plastic zone" and they subsequently close up, it may be assumed that all irreversible work has been confined to areas contiguous with the crack faces. Then the whole area under the load/ extension plot may be attributed to work of fracture. Most of our specimens did close up. However, in the highest percentage PUV coated specimens, it was difficult to propagate cracks at all, and buckling delamination at the back face of the testpiece, or shear deformation along planes perpendicular to the crack in the specimen "arms", terminated the experiments. Hence the 
toughness values at the highest percentage coatings in PUV edge-crack specimens are not known with confidence, but the quoted values if anything are perhaps low.

The toughness data from the Tattersall and Tappin PUV testpieces did not show these effects. They did, however, display a curious behaviour, with the toughness values levelling off after about $C=0.5$, rather than carrying on rising at large $C$. These effects are discussed later.

Tensile and edge-crack fracture toughness results for both silicone vacuum grease and polyurethane coatings are plotted against $C$ in Figs. 7 and 8 . The tensile data remains at or

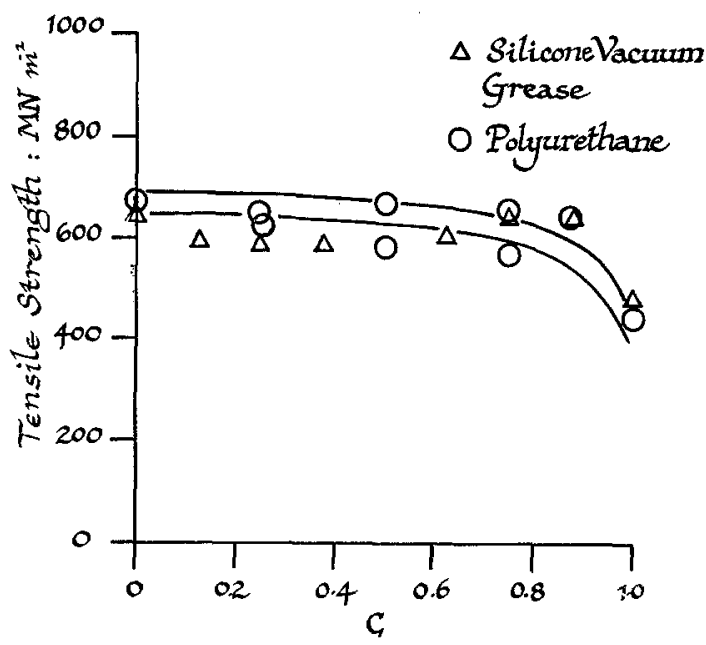

Figure 7 Tensile results for intermittently bonded composites. Full lines represent Equation 4.

about the RoM value until $C$ becomes greater than 0.8 . The fall in $\sigma$ at very large $C$ is anticipated from the analysis in Section 3.

The toughness results with silicone vacuum grease coating fall slightly with increase of $C$, but beyond $C=0.4, R$ becomes modestly greater than the "completely uncoated" (i.e. $C=0$ ) case. The edge crack and Tattersall and Tappin data all essentially agree: for $\nu_{\mathrm{f}}=0.25$, and $C=0, R=45$ to $50 \mathrm{~kJ} \mathrm{~m}^{-2 *}$; for $C=1.0$, $R=60$ to $65 \mathrm{~kJ} \mathrm{~m}^{-2}$.

In contrast, the polyurethane coatings gave marked improvements in toughness for increased percentage coating. In the edge-crack specimens, with $v_{\mathrm{f}}=0.25$, toughnesses about $100 \mathrm{~kJ} \mathrm{~m}^{-2}$ are produced for $C=0.5$, and values around 250 to $300 \mathrm{~kJ} \mathrm{~m}^{-2}$ occur at large $C$. As previously

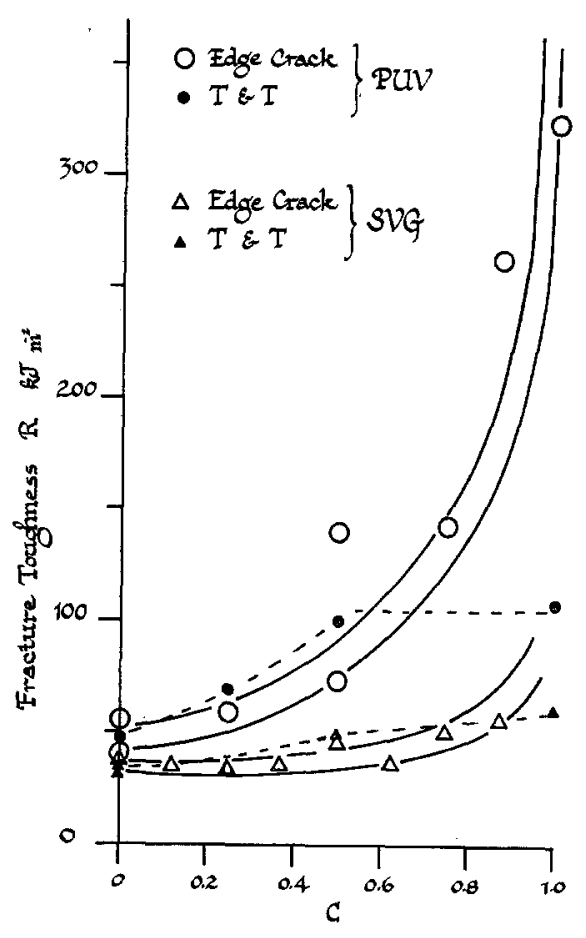

Figure 8 Toughness results for intermittently bonded composites.

mentioned, these latter toughness values may not be precise. The Tattersall and Tappin data levelled out at some $110 \mathrm{~kJ} \mathrm{~m}^{-2}$ for all $C>0.5$, which suggests that one or more components of the toughness in the edge-crack specimens at large $C$, failed to contribute to cracking in the three-point beam bending situation.

To have some feel for the interfacial shear stresses active during pull-out, some of the "used" edge-crack specimens that were partially cracked through were regripped above the original uncracked portion and pulled in tension. After the crack had propagated across the relatively narrow section, and the crack faces had separated, the work subsequently performed in pulling the fibres out was measured from the testing machine chart. Some of the fibres had already been broken in the original edge-crack tests, and the remainder were broken when the crack faces were separated in these pullout measurements. Inspection of the pull-out lengths enabled estimates for $\tau^{\prime}$ to be obtained from the expression for work in pulling out one filament over length $h$, namely $\frac{1}{2} \tau^{\prime} \pi d h^{2}$. The

*The results are different from [1], since larger diameter, stronger, boron filaments have recently been employed, namely 140 instead of $100 \mu \mathrm{m}$ diameter, 3.45 in place of $2.96 \mathrm{GN} \mathrm{m}^{-2}$ for $\sigma_{\mathrm{f}}$; $\mathrm{v}_{\mathrm{f}}$ is also different. 
PUV pull-out lengths were quite long, which helped experimentation.

During pull-out, the interfacial frictional stress for the uncoated samples was about 10 $\mathrm{MN} \mathrm{m}^{-2}$, which should be contrasted with the matrix shear stress of some $70 \mathrm{MN} \mathrm{m}^{-2}$. For most of the PUV coated samples, however, the interfacial shear stress during pull-out seemed to be independent of $C$, having the approximate value of $2 \mathrm{MN} \mathrm{m}^{-2}$. This is perhaps surprising, as one might have expected a frictional stress decreasing with increasing $C$. The SVG data were rather inaccurate, since the pull-out lengths were considerably shorter. Later in this paper, we will see that to obtain agreement between theory and experiment for the toughness data, we would like to have $\tau^{\prime}$ constant for PUV, but varying for SVG.

Finally, to see "how tough" a testpiece could be made, some $v_{\mathrm{f}}=0.5, C=0.8$ tapes were manufactured and laid up into a twelve-layer $4 \mathrm{~mm}$ thick edge-crack specimen, with eight layers perpendicular to the crack line and four layers parallel to the crack. Instead of cracking, the testpiece "yielded" in the arms, giving the warped shape shown in Fig. 9 on unloading. No estimate for fracture toughness is thus available. We can find a bound on it, however, in the following way: Gurney and Hunt [11] and Hahn et al. [12] have shown that generalized

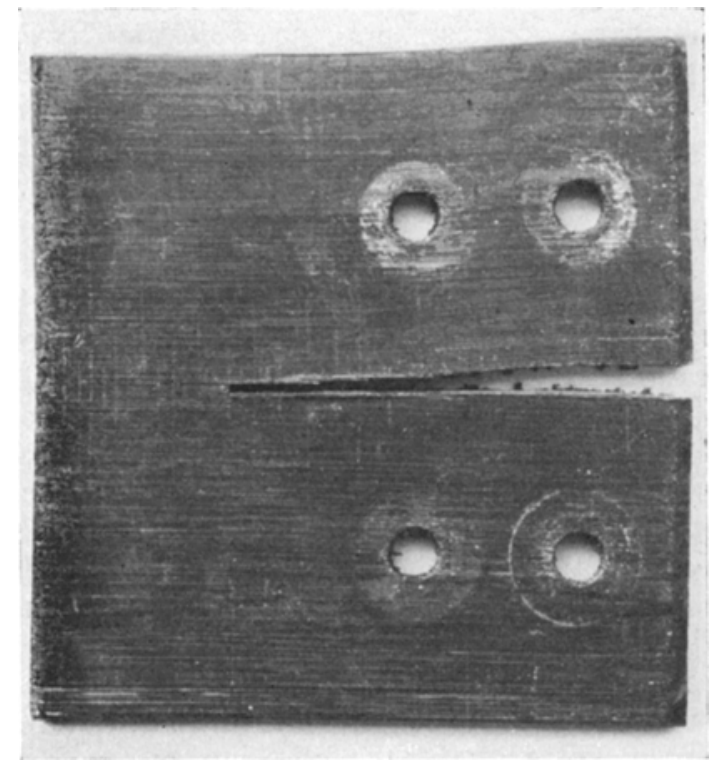

Figure 9 Warped testpiece owing to generalized yielding of arms in high volume fraction, high toughness specimen. yielding in the testpiece, rather than crack propagation, should be expected when

$$
H<\frac{(1.5 \sim 3.0) E R}{\sigma_{\mathrm{y}}{ }^{2}}
$$

where $H$ is the height of the testpiece "arms", and $\sigma_{\mathrm{y}}$ the yield strength. Using the following values with $v_{\mathrm{f}}=0.5$, namely $E=(0.5)(380) \mathrm{GN} \mathrm{m}^{-2}$, $\sigma_{y}=(0.5)(3.45) \mathrm{GN} \mathrm{m}^{-2}$, and inserting $H=38$ $\mathrm{mm}$, we have

$$
R>200 \text { to } 400 \mathrm{~kJ} \mathrm{~m}^{-2} \text {. }
$$

For reference, uncoated specimens with $v_{\mathrm{f}}=0.5$ (such as testpieces made up from as-received Avco Prepreg tape) give $R \sim 100$ to $120 \mathrm{~kJ} \mathrm{~m}^{-2}$.

\section{Discussion}

The tensile strengths of $0.2 v_{\mathrm{f}}$ composites with filaments fully coated with silicone vacuum grease and polyurethane varnish were about 500 and $450 \mathrm{MN} \mathrm{m}^{-2}$ respectively. The "as-received" strength with no coating was some 650 to 700 $\mathrm{MN} \mathrm{m}{ }^{-2}$. For the size of tensile testpieces used, $\psi / n \approx 0.03$ to 0.05 . Applying Equation 4 to the fully coated samples, with $\sigma_{\mathrm{f}}=3.45 \mathrm{GN} \mathrm{m}^{-2}$, $\sigma_{\mathrm{m}}=81 \mathrm{MN} \mathrm{m}^{-2}$ and $d=140 \mu \mathrm{m}$, it would appear that $T$ was about 0.06 for SVG and about 0.05 for PUV. Using these values, Equation 4 has been plotted out versus $C$ in Fig. 7. The general agreement with experimental tensile results at various percentage coatings is reasonable.

The longest pull-out lengths that were observed after completion of the experiments to measure $\tau^{\prime}$ (the interfacial shear stress during pull-out) agreed reasonably well with $\left(l_{\text {crit }}\right)_{\tau_{\text {av }}} / 2$ given by Equation 2 for SVG using $T=0.06$. In the case of PUV, the longest pull-out lengths were consistently greater than $\left(l_{\text {crit }}\right)_{\tau_{\text {av }}} / 2$ with $T=$ 0.05 . If Cook/Gordon debonding were taking place in the intermittently bonded composites, we should expect the pull-out lengths to be longer. At $C=0.25,\left(l_{\text {crit }}\right)_{\tau_{\text {av }}} / 2 \approx 2 \mathrm{~mm}$; with a $25 \mathrm{~mm}$ repeat distance, the Cook/Gordon debond length is some $6 \mathrm{~mm}$ so that the longest pull-out length may be expected to be about 8 $\mathrm{mm}$. This was what we observed, and at the largest percentage coatings, many filaments pulled out the complete "half height" of the specimen (Fig. 10), since the pull-out lengths (incorporating Cook/Gordon debonding) should be greater than $38 \mathrm{~mm}$. Although general agreement for the longest pull-out lengths was obtained, the average pull-out lengths seemed 


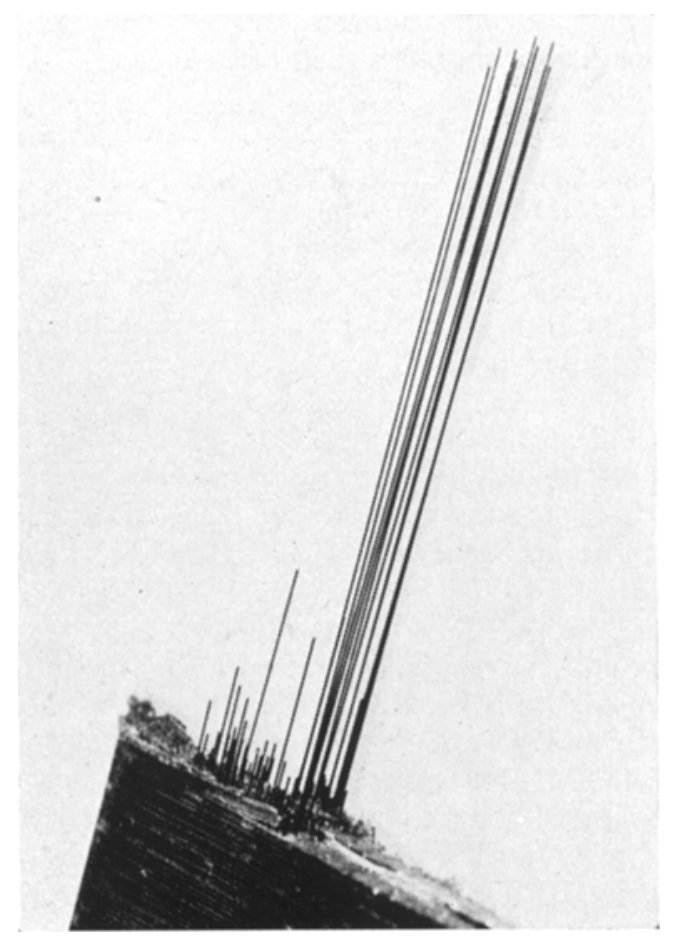

Figure 10 Photograph of pull-out lengths, $38 \mathrm{~mm}$ long. The absence of fibres at the left end reflects failure by local buckling at the "back" of the testpiece.

smaller than $\left(l_{\text {crit }}\right)_{r_{\text {av }}} / 4$ for SVG and smaller than $\left[\left(l_{\text {crit }}\right)_{\tau_{\mathrm{av}}} / 4\right]+\left[C l_{\mathrm{r}} / 2\right]$ for PUV. Nevertheless, the trend of pull-out lengths seemed to indicate that Cook/Gordon debonding was taking place with PUV coatings, but that it was absent with SVG. The marked difference in toughness values (although $T$ was not very different between SVG and PUV), confirms this contention.

Comparison with the equations in the Appendix for the composite toughness is not easy to make without some handwaving about the interfacial toughness values. The matrix toughness itself, $R_{\mathrm{m}}$, may be independently determined from edge-crack or Tattersall and Tappin testpieces: for the epoxy cure cycle that was employed, $R_{\mathrm{m}} \approx 2.5$ to $3.0 \mathrm{~kJ} \mathrm{~m}^{-2}$. The presence of the nylon scrim cloth, in thin edge-crack specimens made up from tapes containing no filaments, had an insignificant effect. In principle, interfacial toughness in mode II can be measured by pulling on embedded filaments, but in practice the experiments are difficult to perform (the pull-out experiments described earlier for the "frictional" $\tau$ ' are, of course, different in nature since the filaments have already broken). Interfacial toughness in mode I does not seem easy to measure. It was argued in [1] that substitution of the matrix toughness for the interfacial toughness was a reasonable approximation in continuously coated composites, because if $R_{\text {if }}\left(R_{\text {if uc }}\right.$ in the intermittent bond context) were greater than $R_{\mathrm{m}}$, matrix material would adhere to the filaments and this was not observed experimentally. A similar postulate is used for intermittently bonded composites where, in addition, the following is suggested for $\rho_{\mathrm{II}}$ : let us assume that the interfacial shear stress is proportional to the mode II stress intensity factor. Then the ratio of coated and uncoated shear stresses $(T)$ becomes

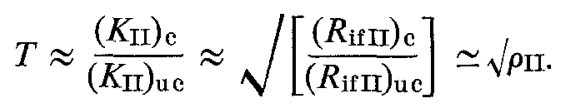

For $T=0.06, \rho_{\mathrm{II}} \rightarrow 36 \times 10^{-4}(\mathrm{SVG})$; for $T=0.05, \rho_{I I} \rightarrow 25 \times 10^{-4}$ (PUV). Equation (A2) is not sensitive to such small values of $\rho$ II over the applicable range of variables, so that it is adequate to consider $\rho_{\mathrm{II}}=0$ in the toughness expressions, or cancel out the $\left[1-C\left(1-\rho_{\mathrm{II}}\right)\right]$ term with the $[1-C(1-T)]$ term (see later).

With that assumption, and with the values of $\tau^{\prime}$ obtained from the pull-out experiments for SVG and PUV, the equations in the Appendix may be plotted and compared with the toughness data. In general terms, it is readily shown that the equations, which incorporate Cook/Gordon debonding, overestimate the observed SVG results, but are in reasonable agreement with the PUV data. There are, however, some questions regarding the precise algebraic formulations of some of the components of the total toughness.

The form of the pull-out contribution relates back to the behaviour of $\tau^{\prime}$ in the pull-out experiments. In the case of PUV, a constant $\tau^{\prime}$ of some $2 \mathrm{MN} \mathrm{m}^{-2}$ seems to be required to describe the toughness results, whereas for SVG a $\tau^{\prime}$ which diminishes with increasing $C$ is required. Such differences in $\tau^{\prime}$ behaviour were broadly observed in the pull-out tests, but the reasons are unclear. Perhaps the interfacial friction stress of the Cook/Gordon debond lengths "biases" the average $\tau^{\prime}$ down to an approximately constant value.

The magnitude of the Piggott/Fitz-Randolph contribution could be one-half of the usually quoted expression, for the following reason. The energy dissipating mechanism is irreversible relative slip between filament and matrix in the 
presence of "full" interfacial bonding. In [2], the situation was modelled where the matrix fracture strain was less than the filament fracture strain, so that filaments were stretched relative to the matrix interface before they fractured (a matrix crack perpendicular to a filament having passed ahead of the filament). Irreversible work is thus performed, and when the filament "sprang back" after fracture, more work was dissipated at the interface. The distance over which slip occurs was shown to be $\left(l_{\text {crit }}\right) / 2$ [2]; the "forward slip" and "back slip" contributions to toughness were the same, and equal to $v_{\mathrm{f}} \sigma_{\mathrm{f}}^{2}\left(l_{\mathrm{crit}}\right) / 6 E_{\mathrm{f}}$, i.e. one-half of Equation A6 as previously quoted. If the filament fracture strain is less than the matrix fracture strain, as is the case for boron/epoxy, presumably the "forward slip" contribution is absent. The curves superimposed on Fig. 8 show both possibilities; the data favour irreversible slip only in one direction.

Thirdly, there is also a question about the magnitude of the Cook/Gordon contribution to toughness. Equation A4 gives values differing by a factor of ten, depending on the magnitude of $\rho_{\mathrm{I}}$, which in turn depends upon the choice of $1 / 5$ or $1 / 50$ for the critical tensile strength ratio (see Appendix). However, the Cook/Gordon component to total toughness itself is comparatively small, so that it is not possible, from the experimental results, to establish which value of $\rho_{\mathrm{I}}$ is appropriate.

It is instructive to present the magnitudes of the relative contributions to $R$ from the various mechanisms, taking into account the foregoing questions. For PUV, with $R_{\mathrm{m}} \approx 2.6 \mathrm{~kJ} \mathrm{~m}^{-2} \approx$ $\left(R_{\text {II if }}\right)_{\mathrm{uc}}, T \approx 0.045, \psi=0.14, \lambda=181, d=140$ $\mu \mathrm{m}, \tau^{\prime}=2 \mathrm{MN} \mathrm{m}^{-2}$ and measuring all toughness values at a crack opening of $h=0.5 \mathrm{~mm}$ and cancelling $\left[1-C\left(1-\rho_{\mathrm{II}}\right)\right] \approx[1-C(1-T)]$, we have from Equations A2, A4, A6 and A11 for $v_{\mathrm{f}}=0.25$,

$$
\begin{aligned}
R= & 2+16+\frac{6.3(0.7 \text { or } 1.4)}{[1-0.955 C]} \\
& +(1.9 \text { or } 19) C^{2}+45\left[\frac{0.14}{(1-0.955 C)}+2 C\right]
\end{aligned}
$$

where $R$ is in $\mathrm{kJ} \mathrm{m}^{-2}$. We see that the contribution from matrix fracture surfaces remains constant at $2 \mathrm{~kJ} \mathrm{~m}^{-2}$; the surfaces contribution from filament debonding also remains constant at about $16 \mathrm{~kJ} \mathrm{~m}^{-2}$, (after the cancelling assumption), the increase in debond area with $C$ being counteracted by a reduction in average $\left(R_{\mathrm{II}} \text { if }\right)_{\mathrm{c}}$. Piggott/Fitz-Randolph stress redistribution increases with $C$, the longer $l_{\text {crit }}$ of higher $C$ giving longer relative slip distances. The Cook/Gordon contribution increases as $C^{2}$, (i.e. $1.9 C^{2}$ or $19 C^{2}$ ) but in total terms is a small contributor to composite toughness. However, the increased debond lengths that accompany the Cook/ Gordon mechanism produce a significant effect on the pull-out contribution, and the latter is an important part of the total toughness.

At $C=1$, we have

$$
\begin{aligned}
R=2+16+(100 & \text { or } 200)+(2 \text { or } 19) \\
& +(141+90)=351 \text { or } 468
\end{aligned}
$$

taking the lower and higher values of the debatable terms. Superimposed in Fig. 8 are two curves given by the foregoing treatment with high and low values as in the example for $C=1{ }^{*}$ We see that in this fully coated case, the Piggott/ Fitz-Randolph contribution is 100 to $200 \mathrm{~kJ}$ $\mathrm{m}^{-2}$ and the pull-out contribution is about 231

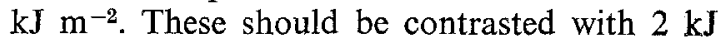
$\mathrm{m}^{-2}$ for the matrix surfaces, $16 \mathrm{~kJ} \mathrm{~m}^{-2}$ from filament debonding, and 2 to $19 \mathrm{~kJ} \mathrm{~m}^{-2}$ from the Cook/Gordon mechanism.

For SVG, with $T=0.06, \tau^{\prime}=3 \mathrm{MN} \mathrm{m}^{-2}$ (varying) and the previous values for the other parameters in Equations A2, A6 and A10 we have

$$
R=2+16+\frac{4.5}{(1-0.94 C)}+10
$$

where half the Piggott/Fitz-Randolph expression is used. The resulting curve for $R$ versus $C$ is superimposed in Fig. 8.

The Tattersall and Tappin toughness results are interesting. In some ways, this testpiece design is not completely satisfactory since there is a transition from plane stress behaviour at the apex of the triangle to plane strain at the base, so that the area under the load/extension curve indicates some ill-defined average toughness. Also, if the triangular section is made by cutting, damaged filaments near the apex also give easy crack initiation. Moreover, the testpiece is strictly unstable, (as may be demonstrated by application of Gurney's crack stability criteria [e.g. 13] to experimental compliance data),

*If the existence of Cook/Gordon debonding is doubted, it might be asked whether Equations A2, A6 and A9 with no Cook/Gordon debonding but with the full Equation A6 for the Piggot/Fitz-Randolph contribution, could satisfy the data. Such an analysis fails to agree with the experiments. 
although with composites those broken filaments bridging the crack faces, after passage of the crack, do introduce a measure of stability through the pull-out contribution to toughness. In the present series of experiments with very tough composites, another effect in such small three-point bending situations came to light.

In the case of SVG, the data essentially agree with the edge-crack results, except that the $C=1$ results are low. For PUV, however, the results are in reasonable agreement with the edge-crack results only up to $C=0.5$; after that, the Tattersall and Tappin data level out at some $110 \mathrm{~kJ} \mathrm{~m}^{-2}$, as opposed to the edgecrack results which continue to increase significantly. It seems as if the pull-out contribution was being limited in some way. Very tough composites have large crack opening displacements before and during crack propagation; some of our beam specimens "bottomed-out" in our three-point bending rig before cracking through, for example. This means that the long pull-out lengths which bridge the crack faces in such circumstances are bent to severe "exposed" radii. Engineers' bending theory suggests that the top fibres in one of our Tattersall and Tappin testpieces, bent to a $5 \mathrm{~mm}$ radius, suffer a stress of some $5 \mathrm{GN} \mathrm{m}^{-2}$. This is greater than the tensile fracture strength of the boron-on-tungsten filaments $\left(\sim 3.5 \mathrm{GN} \mathrm{m}^{-2}\right)$. It would appear that in very tough composites a proportion of fibres break by bending in small beam specimens, thus preventing a full pull-out contribution to toughness. Certainly the pull-out lengths of high $C$ beam specimens seemed short in comparison with those of equivalent tensile or edge-crack specimens. Also, "double filament fractures" have been observed in the high $C$ PUV beam specimens, i.e. where filaments break inside the beam initially, but also break subsequently across the crack faces. Such short filaments may be removed with tweezers after completion of a Tattersall and Tappin test.

It has been pointed out earlier that filaments, which bridge the crack faces for some time after passage of the crack front, stabilize cracking. If some of the pull-out contribution to toughness is lost at large beam deflections by those filaments fracturing in bending, the load/ deflection $R$ locus plots should revert to the natural shape of unstabilized cracks. Fig. 11 shows that the shape of the Tattersall and Tappin load/deflection plots at large displacements in high $C$ PUV specimens are different 828 from the corresponding shape at low $C$.

Since the diameters of typical graphite filaments are much smaller than boron-ontungsten filaments $(\sim 8 \mu \mathrm{m}$ versus $140 \mu \mathrm{m})$, the bending stresses induced at large deflections in Tattersall and Tappin testpieces made of carbon-polyester composites would be much smaller than the filament fracture stress, so that valid toughness data would seem to be obtainable.

The foregoing observations emphasise the care with which the pull-out contribution to toughness must be treated. Although rarely acknowledged in such terms, "strict" fracture mechanics toughness implies displacement reversibility, i.e. crack testpieces return to the origin of load/ displacement plots upon unloading. Pull-out is a frictional contribution to toughness, and unloading the specimen involves additional irreversible work in pushing back "down the holes" those filaments that bridge the crack faces. It can be compared with "reversed plasticity". Pull-out thus has the effect of stabilizing cracking situations that would be otherwise unstable, e.g. in the Tattersall and Tappin testpiece.

It may be a moot point whether pull-out should be included in the basic description of toughness, since the load bearing capacity of a cracked structure could be reduced to negligible values before the full contribution of pull-out were realized. At the same time, pull-out does improve crack stability, which is important. Also, like it or not, pull-out will usually be part of the experimentally measured toughness in typical testpieces, although the actual contribution will vary with testpiece geometry and condition of test. Of importance to experimentalists and designers in the composites field must be the realization that the effective contribution to toughness from pull-out depends markedly on the circumstances, and each case has to be viewed separately.

Further questions regarding valid toughness testing, and associated problems of generalized yielding etc are discussed in a report to NASA [14].

\section{Conclusions}

The intention of this study was to produce high toughness composites without significant loss of tensile strength. This was to be achieved by intermittent coating of the filaments. In brittle fibre/brittle matrix composites as conventionally 


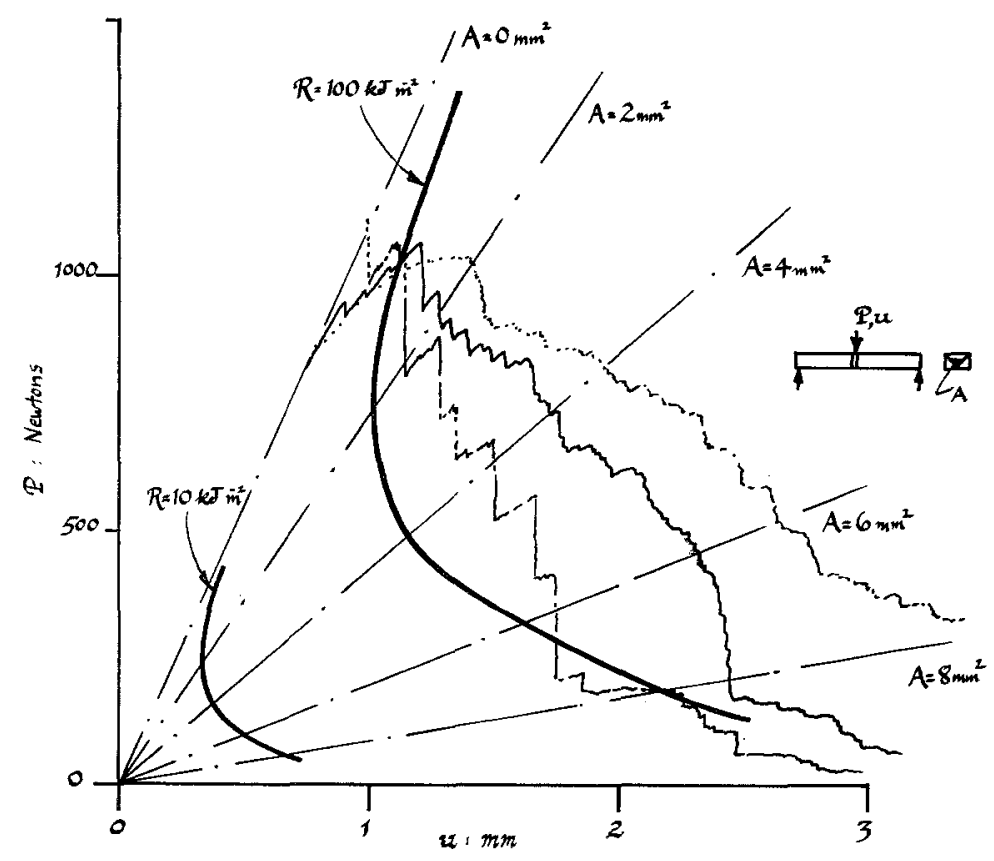

Figure 11 Differences in unstable and stabilized Tattersall and Tappin testpiece toughness loci. Full lines represent theoretical $R$-loci.

made with filaments of uniform interfacial properties, high strengths and high toughnesses are usually mutually exclusive.

Some success has been achieved. For example, unidirectional boron/epoxy composites have been manufactured with toughnesses of over 200 $\mathrm{kJ} \mathrm{m}^{-2}$ while retaining rule of mixtures tensile strengths of some $650 \mathrm{MN} \mathrm{m}^{-2}$. The "asreceived" toughness is some $50 \mathrm{~kJ} \mathrm{~m}^{-2}$ so at least a four-fold increase has been realized. For the volume fractions employed, this seems to represent fracture mechanics stress intensity factors of over $100 \mathrm{MN} \mathrm{m}^{-3 / 2}$ (Young's modulus for boron filaments being about $380 \mathrm{GN} \mathrm{m}^{-2}$ ).

The new concept which has allowed these results is the "intermittent bond", where the special coating process produces alternate regions along the filaments of high and low interfacial shear stresses, and low and high interfacial toughnesses. Then the high tensile strength characteristic of strong interfacial bonding may be combined with the high total fracture toughness of weak interfacial bonding.

Experiments are under way to investigate the mechanical behaviour of cross-ply lay ups as practical structural materials.

Details of the microscopic mechanisms involved in the toughening process are ill-under- stood at present. Marston [6] used a variety of coating materials in his early experiments on intermittently bonded composites; at the time, silicone vacuum grease looked promising. Later, we were prompted to try polyurethane varnish as an intermittent coating material following the "continuously coated" paper by Mullin and Mazzio [15]. The analysis for intermittently bonded composite strength and toughness presented in this paper indicates that interfacial toughness is as significant a property in the overall behaviour as the commonly considered shear strength. The SVG and PUV results seem to indicate that similar interfacial shear strengths may not mean similar interfacial toughnesses, so that the Cook/Gordon mechanism occurs in one system but not the other. Why this should be so is not known.

\section{Appendix. Derivations of toughness components}

(1) $R_{\text {surfaces }}$

$R_{\text {surfaces }}$ consists of components from filament fracture cross-sections, matrix fracture crosssections and surfaces created by interfacial fractures. The filament cross-sectional contribution is negligible in the boron system, and we have, for random filament fracture in 
"untreated" composites [1],

$$
R_{\text {surfaces }}=\left(1-v_{\mathrm{f}}\right) R_{\mathrm{m}}+v_{\mathrm{p}} \cdot \frac{l_{\text {crit }}}{d} \cdot R_{\mathbf{i f}}
$$

where $l_{\text {crit }}$ is the critical length appropriate to the (constant) $\tau$ and $R_{\text {if }}$ is the fracture toughness of the interface between filament and matrix.

For intermittently coated filaments, we may use the fictitious $l_{\text {crit }}$ of Equation 2 in Equation $\mathrm{A} 1$, together with the $\left(R_{\mathrm{if}}\right)_{\mathrm{av}}$ of Equation 3 to give for mode II debonding,

$$
\begin{aligned}
R_{\text {surfaces }} & =\left(1-v_{\mathrm{f}}\right) R_{\mathrm{m}} \\
+ & v_{\mathrm{f}} \psi \lambda\left\{\frac{1-c\left(1-\rho_{\mathrm{II}}\right)}{1-C(1-\mathrm{I})}\right\}\left(R_{\mathrm{ifII}}\right)_{\mathrm{uc}} .
\end{aligned}
$$

Equation $\mathrm{A} 2$ is given as Equation 7 in the main body of text.

It should be mentioned that when the coated length is less than its own critical length, as is often the case in the experiments reported in this paper, the average debond length of coated regions is likely to be the coated length itself and not $l_{\text {erit }} / 4$ which the derivation of Equation A1 strictly assumes; this introduces an error in the use of Equation A2.

\section{(2) $R_{\text {Cook } / \text { Gordon }}$}

Cook and Gordon [5] suggested that if the tensile strength of an interface ahead of a running crack were about $1 / 5$ of the tensile strength of the matrix material, tensile debonding (mode I) would occur at the interface before the crack reached that interface. Gilliland (quoted in [16]) revised the factor to $1 / 50$ on account of anisotropy.

Since "tensile strength" in brittle solids is a reflection of inherent flaw propagation, we may argue that

$$
\begin{aligned}
\frac{\left(\sigma_{\text {tensile interface }}\right)_{\mathrm{c}}}{\left(\sigma_{\text {tensile interface }}\right)_{\mathrm{uc}}} & \approx \frac{\left(K_{\text {Iinterface }}\right)_{\mathrm{c}}}{\left(K_{\text {Iinterface }}\right)_{\mathrm{uc}}} \\
& \approx \sqrt{\left[\frac{\left(R_{\text {if I }}\right)_{\mathrm{c}}}{\left(R_{\text {if I }}\right)_{\mathrm{uc}}}\right]=\sqrt{ } \rho_{\mathrm{I}}}
\end{aligned}
$$

where $K_{\mathrm{I}}$ is the stress intensity factor. For the $\sigma$ ratio to be $1 / 5, \rho_{\mathrm{I}} \rightarrow 4 \times 10^{-2}$; for the ratio of $1 / 50, \rho_{\mathrm{I}} \rightarrow 4 \times 10^{-3}$.

If the filament coating procedure reduces the mode I interfacial toughness by such amounts, Cook/Gordon debonding should occur in "weak" regions ahead of a crack.

If there are $N$ filaments in the plane of the crack, $C N$ will be coated. If Cook/Gordon debonding occurs, the debond length will be about the coated length, in the sense that the 830 mode I crack probably arrests in the adjacent uncoated regions where, presumably, the resistance to cracking in mode $I$ is greater than for the coated region. Then, the debond area is some $\pi d C l_{\mathrm{r}}$ (assuming complete cylindrical debonding, i.e. "behind" filaments in the path of the advancing crack). is

The work absorbed by the $C N$ coated filaments

$C N \cdot \pi d \cdot C l_{\mathrm{r}}\left(R_{\mathrm{ifI}}\right)_{\mathrm{c}}=N \cdot \pi d^{2} \cdot C^{2} \cdot l_{\mathrm{r}} \cdot \rho_{\mathrm{I}}\left(R_{\mathrm{ifI}}\right)_{\mathrm{uc}}$.

But

$$
N=v_{\mathrm{f}} \cdot \frac{4 A_{\mathrm{nom}}}{\pi d^{2}}
$$

where $A_{\text {nom }}$ is the total cross-sectional area in the plane of the crack.

Whence, the Cook/Gordon contribution to toughness is

$$
R_{\mathrm{Cook} / \text { Gordon }}=v_{\mathrm{f}} \cdot 4 C^{2} \cdot \lambda \rho_{\mathrm{I}}\left(R_{\mathrm{ifI}}\right)_{\mathrm{uc}} .
$$

Equation A4 is Equation 8 in the main text. This contribution is appropriate only when

$$
\rho_{\mathrm{I}} \simeq 4 \times 10^{-2} \text { or } 4 \times 10^{-3} \text {. }
$$

For such values of $\rho \mathrm{r}$, Equation A4 gives a comparatively small contribution to toughness; Cook/Gordon debonding does, however, significantly increase the pull-out lengths, and hence the pull-out contribution to toughness.

\section{(3) $R_{\text {redist }}$}

Piggott [2] and Fitz-Randolph [3] gave essentially the following expression for $R_{\text {redist }}$

$$
R_{\text {redist }}=\frac{v_{\mathrm{f}} \sigma_{\mathrm{f}}^{2}}{3 E_{\mathrm{f}}}\left(l_{\text {crit }}\right)=\frac{v_{\mathrm{f}} \sigma_{\mathrm{f}}^{3} d}{6 E_{\mathrm{f}} \tau}
$$

which was the form used in [1] for continuously coated filaments.

For intermittently coated systems, fracture may take place in the coated or uncoated regions. Clearly $R_{\text {redist }}$ is enhanced by the long load retransfer lengths that follow fracture in a coated region.

If we use the average interfacial shear stress given by Equation 1 to represent the overall average behaviour, we obtain from Equation A5,

$$
R_{\text {redist }}=\frac{v_{\mathrm{f}} \sigma_{\mathrm{f}}{ }^{2} \psi \lambda d}{3 E_{\mathrm{f}}[1-C(1-T)]} .
$$

Equation $\mathrm{A} 6$ is Equation 9 in the text. For $C=0$, we regain Equation $\mathrm{A} 5$; for $T=1$, $R_{\text {redist }}$ appropriate to $\left(l_{\mathrm{crit}}\right)_{\mathrm{c}}$ is given. 
The energy dissipating mechanism is irreversible relative slip between filament and matrix in the presence of "full" interfacial bonding, with some occurring before filament fracture and some occurring upon filament "spring back" after fracture. Cook/Gordon debonding should not affect Equation A6, except in so far that the relative slip after debonding would probably be taking place in uncoated regions possessing $\tau_{\text {uc }}$, rather than in some "mixed" coated and uncoated region possessing $\tau_{\text {av }}$. A question is raised in the text about the magnitude of the Piggot/Fitz-Randolph term in those cases where the filament fracture strain is less than the matrix fracture strain.

(4) $R_{\text {pull-out }}$

It was thought by Marston et al. [1] that $R$ pull-out was not significant in the boron-epoxy system. A toughness contribution of some $450 \mathrm{~kJ} \mathrm{~m}^{-2}$ was given by the Cottrell/Kelly equation, which was considerably greater than the total measured toughness of $\sim 35 \mathrm{~kJ} \mathrm{~m}^{-2}$. This suggested that the original interfacial shear strength was not maintained during pull-out. Moreover, electron micrography of fractured boron-epoxy specimens seemed to show that matrix material had relaxed away from the filaments after debonding.

The toughness data for the intermittently bonded composites reported in this paper consistently exceeded the contributions of "surfaces" and "stress redistribution" by significant amounts (although not by the amount that would be given by direct application of the Cottrell/Kelly expression). It must be remembered that the pull-out formula as normally quoted, i.e.

$$
R_{\text {pull-out }}=v_{\mathfrak{f}} \sigma_{\mathrm{f}}^{2} d / 24 \tau
$$

implies complete separation of the severed parts, with the filaments pulling right out. If the crack faces in a toughness test are bridged by filaments upon completion of the measurements, (as is often the case), "full" pull-out is not achieved, and the measured toughness will fall short of predictions. In most experiments the actual pulled-out distance of relative slip between filament and matrix is less than the "average" $l_{\text {crit }} / 4$ for random fracture. Rather, it is of the order of the "crack opening displacement". A modified version of the Cottrell/ Kelly formula should be employed in such cases, based on the actual relative slip between filament and matrix. Although the usual formula was not appropriate, we were wrong to dismiss it completely in [1].

It may be shown that

$$
R_{\text {puli_out }} \approx \frac{2 v_{\mathrm{f}} \tau^{\prime}}{d}\left[2 D h-h^{2}\right]
$$

where $D$ is the average distance from the end of the fractured filament to the plane of gross fracture (Fig. 12) and $h$ is the pulled-out relative

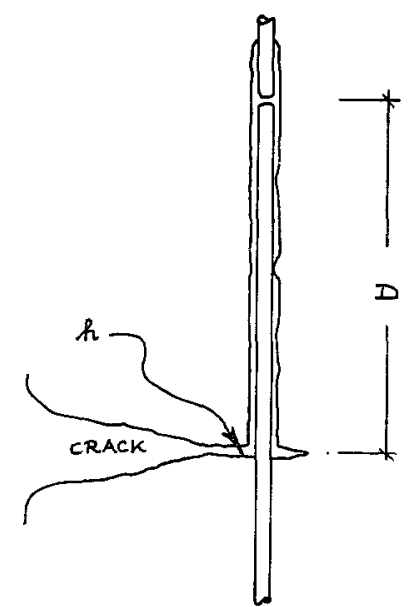

Figure 12 Geometry of pull-out lengths in presence of intermittent bonding.

slip distance. $\tau^{\prime}$ has been written in place of the interfacial shear stress $\tau$ alone, as experiments seem to indicate that a lower "frictional" interfacial traction acts after filament fracture. Putting $D=h=l_{\text {crit }} / 4$ in Equation A8 will not give the usual expression as in Equation A7 because of the integration averaging method used for pull-out [4].

In intermittently coated composites, different $D$ are appropriate for filament fractures in coated or uncoated regions, and also for those cases where Cook/Gordon debonding additionally takes place. Also, the value for the interfacial friction stress $\tau^{\prime}$ has to be known. It was thought that $\tau^{\prime}$ would vary with $C$, in the sense that if the frictional traction during pull-out was perhaps some constant fraction of the interfacial shear stress before debonding, then $\tau^{\prime}$ should decrease with $C$, as $\tau_{\text {av }}$ decreases with $C$ (Equation 1). However, pull-out experiments which attempted to measure $\tau^{\prime}$ suggest d that $\tau^{\prime}$ was constant with one of the coatings, but perhaps varied with the other. Thus, two possibilities are presented in what follows.

In the absence of Cook/Gordon debonding, 
we may use $\left(l_{\text {crit }}\right)_{\tau_{\text {av }}} / 4$ for $D$. Noting that for typical crack opening displacements, (a few $\mathrm{mm}$ ), the $2 h^{2} / d$ term may be neglected, we have from Equation $\mathrm{A} 8$

$$
R_{\text {pull-out }}=\frac{v_{\mathrm{f}} \tau^{\prime} \psi \lambda h}{[1-C(1-T)]} .
$$

If $\tau^{\prime}$ varies according to $\tau_{0}{ }^{\prime}[1-C(1-T)]$, we obtain the simple result that the pull-out contribution to total toughness, measured at the same crack face opening $h$, is constant and equal to

$$
R_{\text {pull-out }}=v_{\mathrm{f}} \tau_{\mathbf{0}}{ }^{\prime} \psi \lambda h .
$$

When Cook/Gordon debonding occurs, $D$ given by $l_{\mathrm{crit}} / 4$ is augmented by an approximate debond length $\mathrm{Cl}_{\mathrm{r}} / 2$, (half the total debond length on one side of the plane of gross fracture). So, neglecting the $2 h^{2} / d$ term,

$R_{\text {pull-out }}$

$$
=v_{\mathfrak{f}} \tau^{\prime} \lambda h\left[\frac{\psi}{[1-C(1-T)]}+2 C\right]
$$

and if $\tau^{\prime}=\tau_{0}{ }^{\prime}[1-C(1-T)]$

$R_{\text {pull-out }}$

$$
=v_{\mathrm{f}} \tau_{0}{ }^{\prime} \lambda h[\psi+2 C[1-C(1-T)]] .
$$

(5) Total toughness

The total toughness of intermittently bonded composites is obtained by adding up the separate appropriate contributions given by the various Equations A2, A4, A6, A9 to 12. For all expressions, with $C=0$, we obtain the formula for total $R$ for continuously coated or uncoated filaments given in [1], with the addition of a pull-out term. This is, of course, the consequence of using the average $\tau$ given by Equation 1 in the uncoated formula of Marston et al. [1].

Note that questions are raised in Section 4 regarding the magnitudes of the different contributions to total toughness.

It should also be noted that Equations A2 and A4 seem to indicate paradoxically that the greatest $R$ would come from the largest values of $\rho$ (i.e. increased interfacial coated toughness). The Cook/Gordon expression however only applies when $\rho$ is very small, and in the case of Equation A2 the implication of large toughness comes about from the fact that, on average, every filament fracture is accompanied by a debond length of $l_{\text {crit }} / 4$, so that if the coated critical lengths are long (because of low $\tau_{\mathrm{c}}$ ), the tougher the interface the better. But some filament 832 fractures will have zero debond length, and these are the ones that set up energetic matrix cracks which clearly will not be arrested by tough interfaces in their path.

\section{Acknowledgements}

Dr T. U. Marston's thesis work provided the stimulus for the study reported here, and I am grateful to him for helpful discussions. Thanks are owed to Messrs W. H. Durrant, J. Allen and G. Streelman who gave great help with the experimentation. Most of the work was performed under NASA Grant NGR 23-005-528 and I take pleasure in acknowledging the guidance given by $\mathrm{Dr}$ W. B. Fichter at the Langley Research Center.

The author is grateful to the referee of this paper for pointing out that when glass fibres are silane-coated for use in resins for GRP, electron microscope studies show the coatings to be laid down not uniformly, as might be expected, but irregularly with isolated islands of the coupling agent. This result is similar to the model described here.

\section{References}

1. T. U. MARSTON, A. G. ATKINS and D. K. FELBECK, J. Mater. Sci, 9 (1974) 447.

2. M. PIGGOTT, ibid 5 (1970) 669.

3. P. W. R. BEAUMONT, J. FITZ-RANDOLPH, D. C. PHillips and A. S. TEtelman, J. Comp. Mater. 5 (1971) 542; see also J. Mater. Sci. 7 (1972) 289.

4. A. KELLY, in "Strong Solids" (Oxford University Press, 1966).

5. J. CoOK and J. E. GORdon, Proc. Roy. Soc. (Lond.) A282 (1964) 508.

6. T. U. MARston, Ph.D. Dissertation, University of Michigan (1973).

7. J. Mullin, J. M. Berry and A. Gatti, J. Comp. Mater. 2 (1968) 82.

8. A. G. ATKINS, Letters to Nature 252 (1974) 116.

9. H. G. TAtTersall and G. TAppin, J. Mater. Sci. 1 (1966) 296.

10. ASTM Special Technical Publication No. 463, 1970.

11. C. GURney and J. Hunt, Proc. Roy. Soc. (Lond.) A299 (1967) 508.

12. G. T. HAHN, M. SARRATE and A. R. ROSENFIELD, Int. J. Fract. Mech. 7 (1971) 435.

13. C. GURNEX and Y. W. MAI, Engr. Fract. Mech. 4 (1972) 853 .

14. A. G. AtKins, Final Technical Report to NASA, August 1974.

15. J. v. Mullin and v. F. Mazzio, J. Mech. Phys. Solids 20 (1972) 391.

16. A. Keldy, Proc. Roy. Soc. (Lond.) A319 (1970) 95.

Received 23 September and accepted 20 November 1974. 